\title{
Sensor Data Collection System for Health Monitoring of Railway Structures
}

\author{
Yoshihiro WATANABE \\ Researcher, \\ Facilities Management Systems Laboratory, Transport Information Technology Division
}

Hiroshi HAYA, Dr. Eng.

JR East Consultants Company (on assignment from Foundation \& Geotechnical Engineering Laboratory, Structures Technology Division)

\begin{abstract}
Masahiro SHINODA, Ph.D.
Assistant Senior Researcher,

Hirokazu OOMURA

Researcher,

Foundation \& Geotechnical Engineering Laboratory, Structures Technology Division

Tatsuya NIHEI

Hokkaido Railway Company (on assignment from Concrete Structures Laboratory, Structures Technology Division)

Norio SATO

Senior Researcher,

Ryuji TSUCHIYA

Senior Researcher, Laboratory Head, Facilities Management Systems Laboratory, Transport Information Technology Division

The application of a health-monitoring system to railway structures is currently under discussion, and several prototype systems have already been implemented and tested. Those systems, often referred to as health-monitoring systems, are expected to have a positive effect on maintenance for structures. We have developed a health-monitoring system in which two methods of data collection can be selected: one uses RFID tags and a PDA (Personal Digital Assistant) and the other uses a wireless radio network (ZigBee, IEEE 802.15.4) and a cellular phone network. This paper gives an overview of our sensor data collection system and its application to railway structures.
\end{abstract}

Keywords: wireless sensor network, maintenance for structures

\section{Introduction}

Railway facility maintenance is an essential part of ensuring safe train operation. Maintenance personnel usually inspect facilities visually, which is a labor-intensive and time-consuming process. Furthermore, their judgment of the soundness of each facility is mainly based on heuristics skills acquired over time, but there is now a lack of inspectors with the required level of experience. We therefore need to establish a new framework that enables maintenance personnel to inspect facilities in a more efficient and less labor-intensive manner.

Accordingly, we have developed a sensor data collection system to monitor the condition of railway structures such as viaducts and tunnels [1]. In this system, we can select an appropriate way to collect data depending on deformation of structure: by RF-ID tag or lowpower radio. Data collected using low-power radio are stored in a relay station, and are transmitted to the maintenance office using a cellular phone network.

The most important issue to be addressed in radio transmission is the reduction of energy consumption. The system applies low-power radio in which energy consumption has been reduced by minimizing the frequency of sensing and transmission. However, regardless of the system's energy-aware behavior, data are transmitted immediately to the maintenance office when a sensor records a value that exceeds the predefined threshold level. This system can cope with damage to RC frames and foundation structures as well as cracks in tunnels. The data collected can be easily referred to and analyzed by the management district using a PC. The main functionalities of the system are data reference, data registration, database master maintenance and data analysis. Its application enables the streamlining of railway structure inspections and the improvement of inspection precision.

\section{Sensor data collection methods}

The application of a health-monitoring system to railway structures is currently under discussion, and a number of experimental systems have already been implemented and tested. It is expected that health-monitoring systems will have a positive effect on maintenance for structures. We have developed a health-monitoring system in which two methods of data collection can be selected: one uses RFID tags and a PDA (Personal Digital Assistant) interface, and another uses a wireless radio network (ZigBee [2], IEEE 802.15.4) and a cellular phone network. 


\subsection{Collecting sensor data using RFID tags}

The RFID tags adopted in this system are the passive type in which a tag reader supplies electronic power electromagnetically. We use $125 \mathrm{kHz}$-frequency radio waves, which are less vulnerable to the effects of water and metal materials. Figure 1 shows a photo of a damage-detecting sensor, which consists of three strain-measuring gauges and an RFID tag. The gauges and the tag are connected by lead wires. Figure 2 shows a photo of the RFID tag reader and the PDA. They are connected by an RS-232C cable, and the data from the three gauges can be imported into the PDA using electric power supplied by the tag reader.

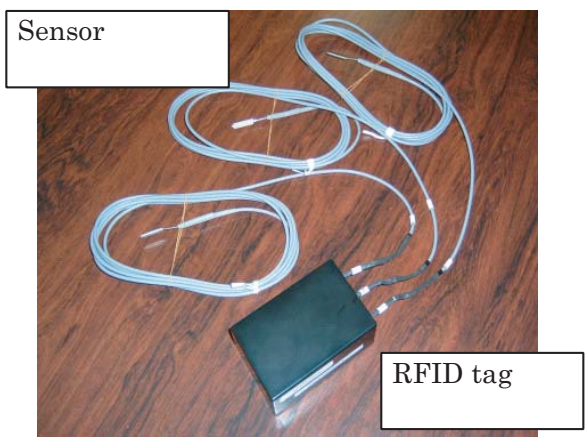

Fig. 1 Damage-detecting sensor

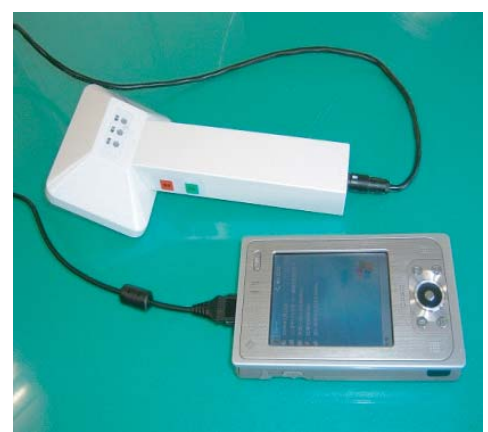

Fig. 2 RFID tag reader and PDA

\subsection{Investigation of radio transmission performance}

The locations where sensors are distributed make it difficult to charge batteries frequently, meaning that the energy consumption of the sensors must be sufficiently low. Table 1 shows the specifications of the radio devices selected as candidates for our health-monitoring systems. Figure 3 shows the throughput of the data transmission for each device.

Interference with wireless LAN (IEEE $802.11 \mathrm{~b} / \mathrm{g}$ ), which is commonly used along railway lines, can be a problem when using wireless technologies in railway

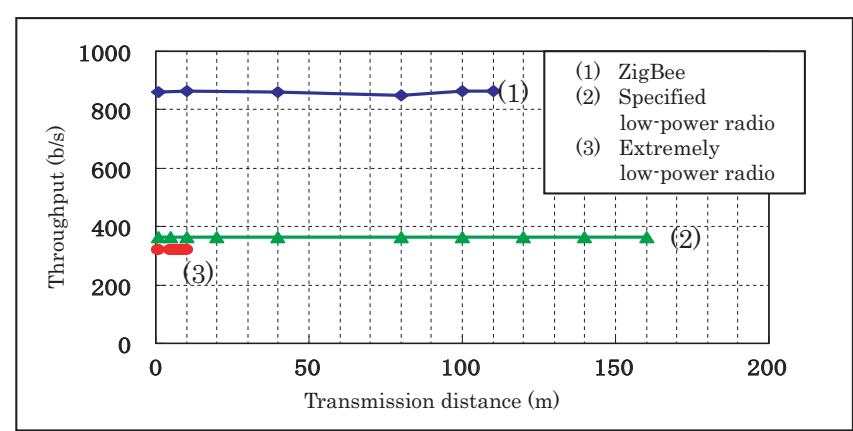

Fig. 3 Transmission throughput

environments. However, the test results revealed that there is no interference as long as the carrier-to-noise ratio $(\mathrm{C} / \mathrm{N}$ ratio) for receiving is greater than $10 \mathrm{~dB}$. Another method to avoid interference with wireless LAN is to use a channel different from that used by the wireless LAN. After transmitting data, ZigBee radio devices go into sleep mode, so undesirable effects on wireless LAN can be minimized. Since the device does not spend much time actually transmitting, data can be sent while the wireless LAN is inactive. It should also be pointed out that the ZigBee radio device is capable of multi-hop data transmission even if it cannot transmit data directly to the destination. Taking these radio wave and data transmission characteristics into account, we decided to use ZigBee radio for data transmission. Figure 4 shows the radio device for a peak sensor, and Fig. 5 shows the device for a $\pi$-shaped displacement-measuring sensor. Radio devices periodically receive data from sensors by battery power and transmit data to relay stations (Fig. 6 ). The relay stations are operated by solar battery power

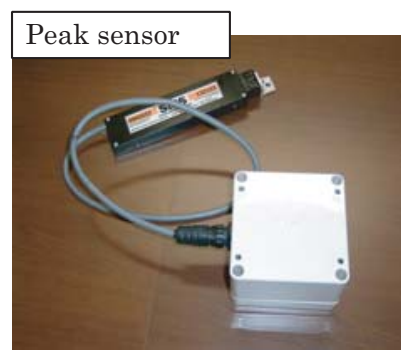

Fig. 4 Radio device for peak sensor

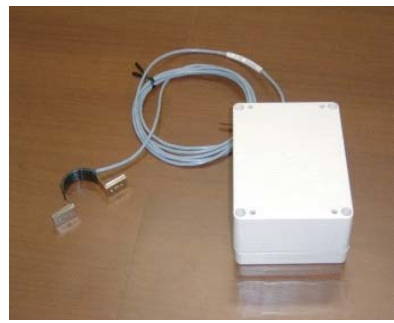

Fig. 5 Radio device for $\pi$-shaped sensor

Table 1 Specifications of low-power radio devices

\begin{tabular}{|l|c|c|c|c|}
\hline \multicolumn{1}{|c|}{ Radio device } & Frequency band & $\begin{array}{c}\text { Transmission distance } \\
(\mathrm{m})\end{array}$ & $\begin{array}{c}\text { Transmission speed } \\
(\mathrm{kbps})\end{array}$ & $\begin{array}{c}\text { Power consumption } \\
(\mathrm{mW})\end{array}$ \\
\hline ZigBee & $2.4 \mathrm{GHz}$ & 30 & 250.0 & 60 \\
\hline $\begin{array}{l}\text { Specified } \\
\text { low-power radio }\end{array}$ & $429 \mathrm{MHz}$ & $30-300$ & 2.4 & 59 \\
\hline $\begin{array}{l}\text { Extremely } \\
\text { low-power radio }\end{array}$ & $315 \mathrm{MHz}$ & $30-200$ & 2.0 & 66 \\
\hline
\end{tabular}




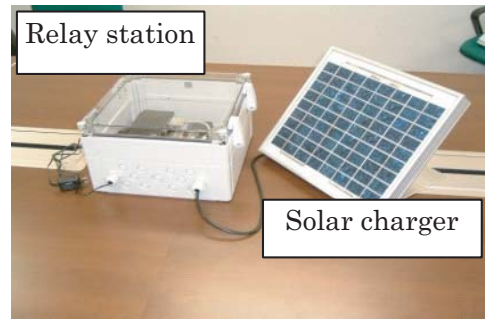

Fig. 6 Relay station

or a $100-V$ DC power supply, and transmit data to a database server located in the branch office of the railway operator periodically (or when data stored in the relay station reaches a predefined amount) using a cellular phone network.

\subsection{Intelligent relay station}

The important issues in radio transmission are the electric power supply and the data transmission throughput. In our system, power consumption is greatly reduced by adopting low-power radio transmission and optimized operation of sensor devices. For example, when relay stations are not sensing and/or transmitting data, they fall into sleep mode and thus consume very small amounts of energy. The volume of transmitted data is also reduced through data compression and the removal of duplicated data (i.e., if data to be sent happens to be the same as the previous transmission, it will be suppressed). When critical data is gathered, the system immediately transmits it regardless of the energy saving behavior of the relay stations described above. The system also monitors battery voltage so that notification of any reduction in residual capacity in the battery will be sent before the functionalities of relay stations (such as data transmission) are extensively disrupted.

\subsection{Reference of collected data}

Collected data is registered on the administration center server, and maintenance officers can refer to the data on a PC in the maintenance office at any time. The main programs of this system are data reference, data registration, database master maintenance and data analysis.

1) Data reference program

Using this system, maintenance officers can refer to collected sensor data and the specifications of structures that sensors are attached to. The specifications of structures and pictures/drawing of them, etc. can be referred to using rough drawings (Fig. 7) or through a conditional search (Fig. 8).

2) Data registration program

Data collected using RFID tags are registered into the database by connecting the PDA to a PC. Data collected through radio transmission are directly registered into the database via a cellular phone network and network server.

3) Database master maintenance program

This program updates, adds and deletes master information on the specifications of structures, sensor types

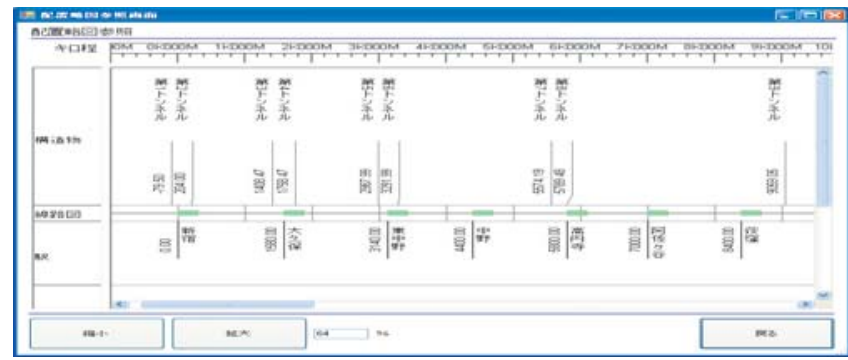

Fig. 7 Rough drawings of structures

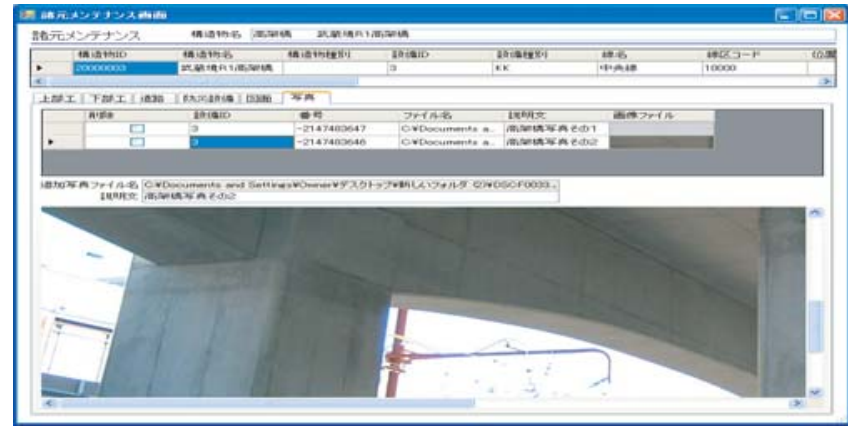

Fig. 8 Reference of specifications

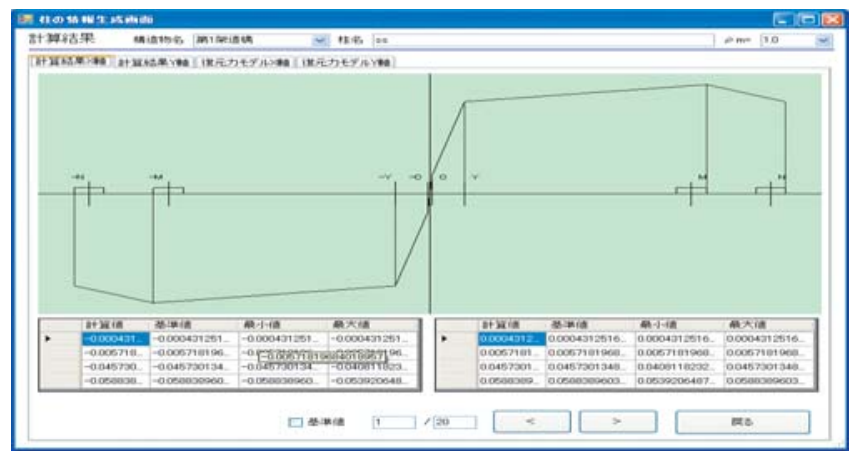

Fig. 9 Calculation results based on the restoration model

and measurement details as well as sensor locations.

4) Data analysis program

This program carries out time-series analysis using measured data registered in the database. Based on the data collected from sensors, it evaluates the level of damage to $\mathrm{RC}$ rigid bridges using the restoration model already developed (Fig. 9).

\section{Application to civil engineering structures}

This chapter outlines the three types of sensor applied to bridges and tunnels.

\subsection{Application to damage-level detection for rigid- frame bridges}

$\mathrm{RC}$ rigid-frame bridges are designed to be robust enough to withstand large earthquakes. Since piers are particularly prone to tremor-related damage, they are subjected to extra inspections by maintenance workers who monitor and check structures carefully after earthquakes occur. 
Recently, reinforcement using steel plates (i.e., rolling steel plates on piers) is enforced on many piers to offer continuous resistance. In such cases, it is difficult to identify damage through simple visual inspection of structures.

We have already established knowledge of the relationship between the maximum slant and damage to piers during earthquakes. Measuring the maximum slant of piers therefore enables the related damage to be checked immediately. This will lead to increased efficiency in restoration work and a reduction in the length of time that train operation is suspended after earthquakes.

(1) Sensor data collection with RFID tags

As shown in Fig. 10, a slant-measuring device is placed on a viaduct, and a measuring stick follows the earthquake response of the piers. The jig, which is vertically set at the measuring point, mediates between the piers and the sensors. Areas where damage tends to be concentrated are avoided as locations for measuring points. Sensors can measure the maximum displacement in both the positive and negative directions and record peak values. This sensor, which does not use battery power, demonstrates high durability and is relatively inexpensive. Pairs of sensors are installed at each point - one parallel to the rails, and the other at right angles to them. The direction of pier movement during earthquakes can be arbitrary, so a jig capable of extracting displacement in a particular direction is attached to the peak sensor and touches the measuring stick. Using this technique enables measurement of the maximum earthquake response displacement in both directions at the same time. The maximum angle data is important in detecting the level of damage. The maximum displacement seen at the measuring point by the inclination of the measuring stick is ascertained using the peak sensor. The maximum angle of earthquake response is calculated using this maximum displacement value and the distance between the fulcrum and the measuring point. The peak sensor is installed in the upper zone of the piers of the RC rigid frame. In order to obtain sensor data via RFID tags located in the lower zone of the piers, peak sensors and RFID tags are connected by lead wires (Fig. 11). Sensor data is read using a tag reader and a PDA (Fig. 2). Figure 12 shows the measurement viewer screen on a PDA. The sensor data consists of the maximum and minimum values in the $\mathrm{X}$ and $\mathrm{Y}$ directions. Data stored on the PDA are registered to the database server when maintenance workers return to the maintenance office.

(2) Sensor data collection by radio transmission

Using two radio devices (Fig. 4) as damage level detection nodes, the angle data mentioned previously in the previous paragraph are transmitted via a relay sta-
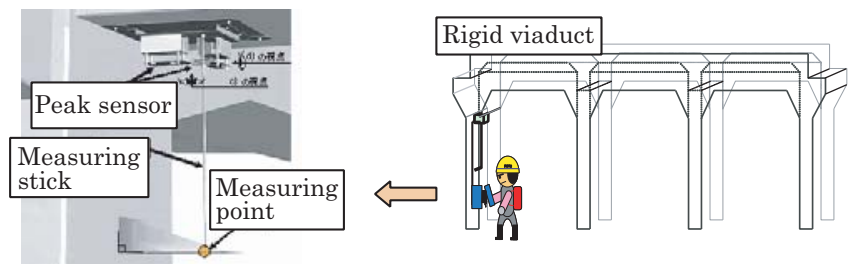

Fig. 10 The slant-measuring device and its installation

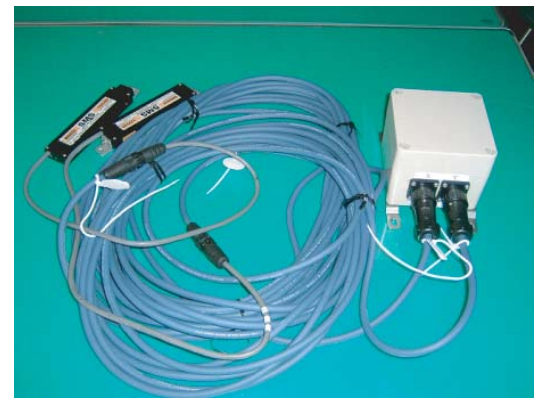

Fig. 11 Peak sensors and RFID tags

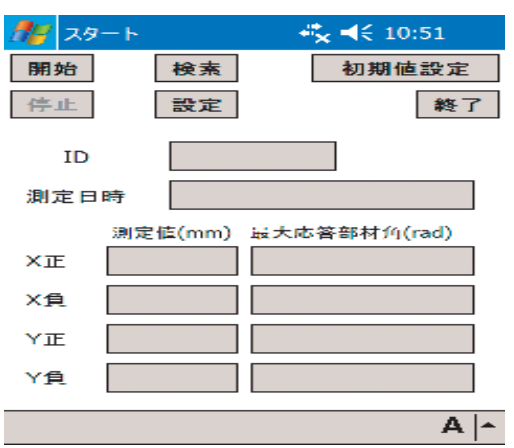

Fig. 12 PDA screen for measuring

tion (Fig. 6) to the database server in the administrative area. Data measurement cycles for each node and data transmitting cycles from the relay station to the database can be set arbitrarily.

\subsection{Damage self-detection for underground parts of viaducts}

The foundations of structures are usually built underground, and the substructure of bridge piers located in running water are submerged, making it difficult to check the condition of such structures visually. Since the foundations or substructures of rigid frame bridges (a typical structure used in railways) are also underground, they are likewise difficult to check visually. In order to ascertain damage caused by earthquakes or other external forces in such structures, maintenance workers carefully inspect the underground parts visually. If there is any possibility of deformation in these parts, nondestructive inspection is carried out, or the underground part of the pier is excavated for visual inspection. For safety reasons, severe restrictions are placed on excavation during train operating hours. As a result, the work must be carried out within a short time during the night when no trains are running, which is a very difficult task.

To support inspection and diagnosis of civil engineering structures for which visual checking is difficult, we have developed a self-checking system for underground structures. Figure 13 illustrates the installation of this system.

As can be seen from Fig. 13, a damage-detecting sensor is set inside the pier at the point susceptible to damage. A lead wire connects this sensor to an RFID tag installed inside the pier above ground level.

Maintenance workers collect sensor data via the RFID tag by radio transmission using a tag reader that 


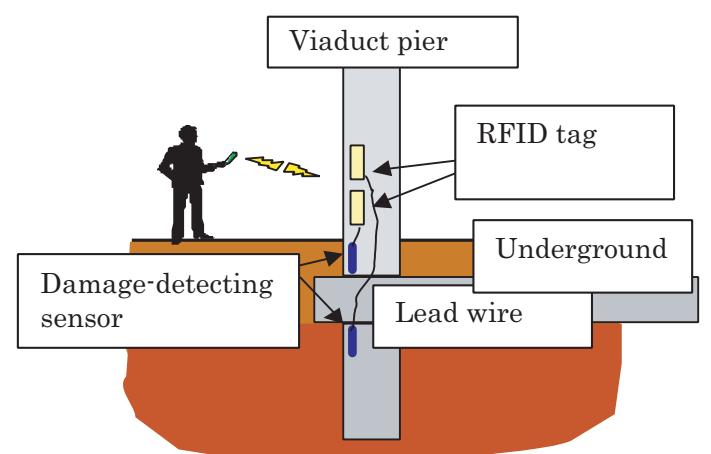

Fig. 13 Installation of damage-detecting sensor

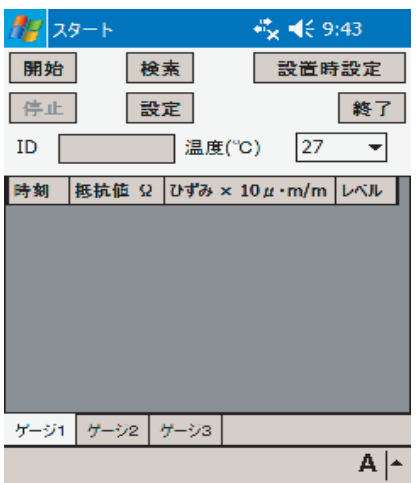

Fig. 14 PDA screen for reading data

can also be used for RC rigid frames. Figure 14 shows the PDA screen during data reading. The system can also be effectively applied to the inspection of structures in passenger stations. In these areas, the presence of shops means that piers tend to be covered with ornamental walls; this system enables the collection of data on pier condition without removing the walls.

\section{Collecting sensor data through ground-to-train wireless communication}

\subsection{Advantage of ground-to-train wireless communi- cation}

There are a number of methods for collecting data from sensors attached to a variety of railway structures. As described in the previous sections, RFID tags can be used to relay sensing data to PDA. With this method, maintenance workers must travel regularly to all locations where sensors are installed. This is a labor-intensive task, and can be very costly. In some cases, an ad-hoc wireless sensor network can be constructed along railway lines to enable multi-hop transmission of sensing data to data sinks (i.e., locations where access to conventional data networks is available). However, the installation and maintenance of sensor networks can be very costly, and deploying this kind of network to cover all railway lines is unrealistic.

We therefore propose a sensing data collection system that uses ground-to-train wireless communication. When a train comes across a location in which sensors are attached to railway structures, it receives sensing data from the sensors by wireless communication technology and transports them to one of the maintenance offices. This method can greatly reduce costs, although maintenance workers must accept a certain level of latency in the data collection. In the next section, we describe the results of testing carried out to evaluate the feasibility of this method.

\subsection{Test results}

We carried out a test to ascertain whether moving objects running at the same speed as a conventional train (i.e., not a Shinkansen train) in service can receive sensor data properly.

In the test, we installed a transmitter for the specified low-power radio on the ground to send one packet message containing six bytes of data every two seconds. The data were collected by a receiver installed on the back of the driver's seat in a car (Fig. 15). Figure 16 shows the relationship between the number of successful receipts of the message and the driving speed, and Fig. 17 shows the relationship between the number of successful receipts and the transmission distance.

As can be seen in Fig. 16, as the speed of the car increases, the number of successful receipts decreases. Data were successfully received even at a driving speed of $125 \mathrm{~km} / \mathrm{h}$. With the increase of the car speed, the communication time between the transmitter on the ground and the receiver installed in the car decreases, and the number of successful receipts also decreases. As Fig. 17 shows, the rate of successful receipt does not depend on the transmission distance as long as it is less than $20 \mathrm{~m}$.

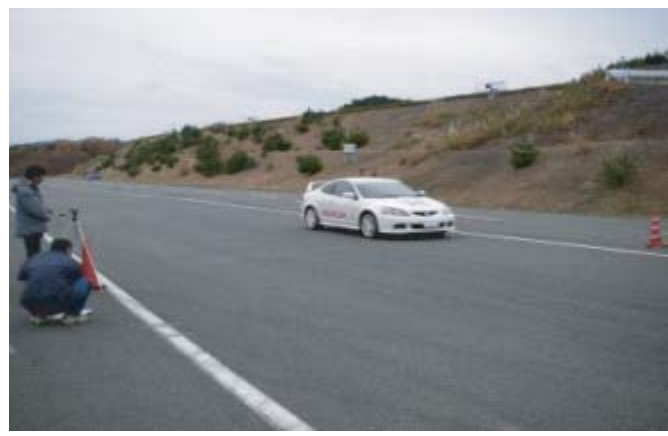

Fig. 15 Field test

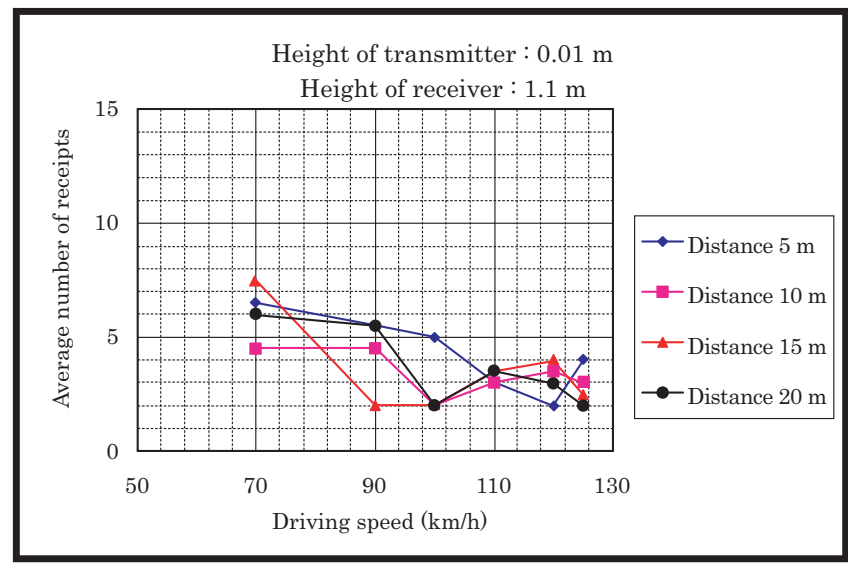

Fig. 16 Number of successful receipts (1) 


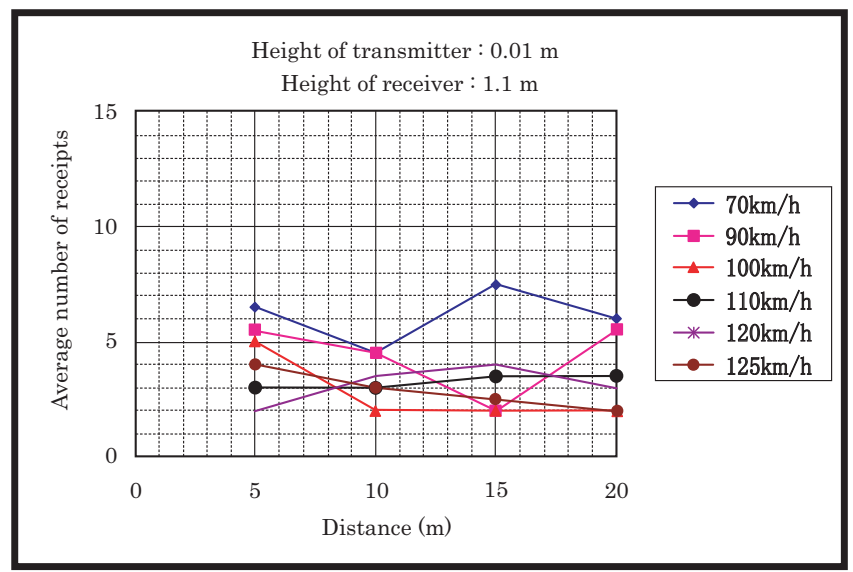

Fig. 17 Number of successful receipts (2)

We therefore conclude that if the size of the data to be sent is sufficiently small, a train moving at $125 \mathrm{~km} / \mathrm{h}$ can successfully collect sensor data as it runs along railway lines.

\section{Future works}

We have developed a health-monitoring system for railway structures using cutting-edge information technology. Figure 18 shows the grand design of the whole system eventually targeted. Currently, a number of issues need to be resolved in order to complete the system. These include: (1) the development of highly reliable sensors with a low failure rate, (2) the realization of solid waterproof performance and corrosion prevention (as sensors are placed along railway lines in environments where the soil has a high water content), (3) the determination of threshold values for emergency alarms, and (4) the development of an efficient and stable power supply system.

We plan to continue our research in the future to solve these problems. We hope to be able to put the system to practical use to meet the needs of railway companies, which have to manage large numbers of civil-engineering structures.

\section{Acknowledgment}

This research was conducted using a subsidy from the Ministry of Land, Infrastructure, Transport and Tourism of Japan.

\section{References}

[1] Sato, N., Nihei, T., Isono, J., Nakayama, T., Watanabe, Y., "Development of a Sensor Data Collecting System for Health Monitoring of Railway Structures," RTRI Report, Vol. 22, No. 6, pp. 35 - 40, 2008 (in Japanese).

[2] ZigBee Alliance, http://www.zigbee.org/.

[3] Crossbow Technology, "Crossbow Technology Wireless Sensor Networks Overview," http://www. xbow.com/Technology/Overview.aspx.

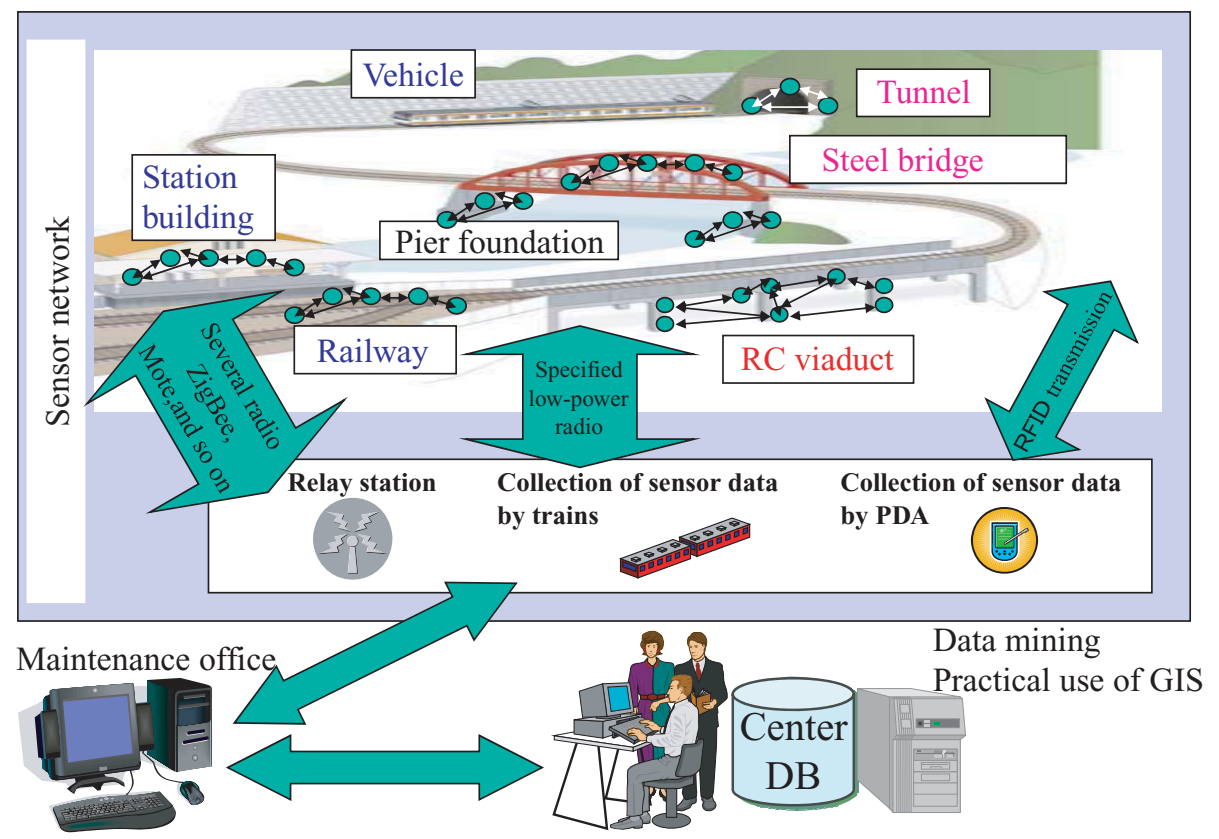

Fig. 18 Grand design of the whole system 